\title{
X-ray absorption spectroscopy study of $\mathrm{Cu}(\mathrm{II})$ coordination in the interlayer of montmorillonite
}

\author{
Sung Pil Hyun ${ }^{1,2, *}$ and Kim F. Hayes ${ }^{1}$ \\ ${ }^{1}$ Department of Civil and Environmental Engineering \\ University of Michigan \\ Ann Arbor, MI 48109, USA; \\ ${ }^{2}$ Geologic Environment Division \\ Korea Institute of Geoscience and Mineral Resources \\ Yuseong-gu, Daejeon 305-350, Korea
}

\begin{abstract}
Cu}$ (II) coordination in the interlayer of an expandable clay mineral montmorillonite is studied using X-ray absorption spectroscopy (XAS) along with electron paramagnetic resonance (EPR) and X-ray diffraction (XRD). Ab initio calculations are performed using FEFF code to reproduce the X-ray absorption near edge structure (XANES) and extended X-ray absorption fine structure (EXAFS) portion of the experimentally measured XAS spectra. $\mathrm{Cu}(\mathrm{II})$ coordination changes depending on the $\mathrm{Cu}$ (II) loading and hydration state of the interlayer. XRD shows that the $\mathrm{Cu}$-saturated montmorillonite has the $d_{001}$-spacing values corresponding to the interlayer thickness of four and one water layer(s) for the hydrated and dehydrated interlayer,

\footnotetext{
*Corresponding author: Dr. Sung Pil Hyun

Current Address: Korea Institute of Geoscience and Mineral Resources

124 Gwahang-no, Yuseong-gu

Daejeon 305-350, Korea

Tel) $+82-42-868-3315$

Fax) +82-42-868-3414

E-mail) sphyun@kigam.re.kr 
respectively. EPR shows that $\mathrm{Cu}(\mathrm{II})$ in the fully hydrated, unsaturated interlayer behaves similar to free $\mathrm{Cu}(\mathrm{II})$ ion in a bulk aqueous solution, while $\mathrm{Cu}(\mathrm{II})$ forms a square planar complex in the dehydrated interlayer. $\mathrm{Cu}(\mathrm{II})$ in the fully hydrated, $\mathrm{Cu}$-saturated montmorillonite has a characteristic singlet $1^{\text {st }}$ derivative XANES spectrum. FEFF calculations show that this singlet feature originates from a quasi-regular octahedral coordination of water molecules around the interlayer $\mathrm{Cu}(\mathrm{II})$ atom. All other samples and model compounds including the dry $\mathrm{Cu}$-saturated montmorillonite, wet and dry unsaturated montmorillonite, aqueous $\mathrm{Cu}(\mathrm{II})$, cupric nitrate salt $\left(\mathrm{Cu}\left(\mathrm{NO}_{3}\right)_{2} \cdot 4.5 \mathrm{H}_{2} \mathrm{O}\right)$, and $\mathrm{Cu}(\mathrm{II})$ hydroxide precipitates have doublet $1^{\text {st }}$ derivative XANES spectra. FEFF calculations suggest that the doublet features arise from an axially elongated octahedral coordination under the Jahn-Teller effect or square planar coordination. FEFF calculations of the EXAFS spectra as a function of the axial oxygen bond length demonstrate that a destructive interference between backscattering from equatorial oxygen $\left(\mathrm{O}_{\mathrm{eq}}\right)$ and that from axial oxygen $\left(\mathrm{O}_{\mathrm{ax}}\right)$ atoms leads to an apparent coordination number $(\mathrm{CN})$ less than six expected for the tetragonal coordination, with the farther, loosely bound axial oxygen atoms making a minor, yet negative contribution to the $\mathrm{CN}$ determined by the EXAFS analysis. This study shows that $\mathrm{Cu}(\mathrm{II})$ has interchangeable octahedral, tetragonal, and square planar coordination in the interlayer of montmorillonite, depending on $\mathrm{Cu}$ (II) loading and degree of hydration. The quasi-regular octahedral coordination of the interlayer $\mathrm{Cu}(\mathrm{II})$ in montmorillonite is a new finding of this study.

Key Words: interlayer $\mathrm{Cu}(\mathrm{II})$; $\mathrm{XAS}$; montmorillonite; octahedral coordination; tetragonal coordination; square planar coordination 


\section{Introduction}

Copper input to soil environments has been increasing from anthropogenic sources such as mining activities, agricultural applications as antibiotics and fertilizers, and industrial wastes from electrical industries, in addition to natural sources largely from the chemical weathering of Cu-containing rocks (Bhattacharyya and Gupta, 2006; El-Bayaa et al., 2009; Veli and Alyuz, 2007). As an essential element for life, its toxicity as well as deficiency may cause acute and chronic problems to humans, animals, plants, and micro-organisms (Yruela, 2009; Ryan et al., 2014; Flemming and Trevors, 1989). Clay minerals play an important role as a natural scavenger of copper in controlling its mobility and fate in soil environments (Alvarez-Puebla et al., 2004; Schlegel and Manceau, 2013; Brigatti et al., 2004).

The coordination structure of copper(II) aqua ions has been experimentally studied using diffraction methods such as X-ray and neutron diffraction. These techniques supported the widely accepted $\mathrm{Cu}(\mathrm{II})$ coordination model of an axially elongated octahedral coordination with unusually fast water exchange at the axial oxygen ligands in aqueous environments due to the $3 d^{9}$ electronic structure and consequent Jahn-Teller distortions (Richens, 1997).

Extended X-ray absorption fine structure (EXAFS) spectroscopy has been also applied to the structural studies of $\mathrm{Cu}(\mathrm{II})$ adsorption complexes on clay and oxide minerals as well as aqueous $\mathrm{Cu}(\mathrm{II})$. However, the curve fitting involved in the data analysis has often led to structural parameters contradicting the existing body of knowledge on the local coordination environment of the adsorption complexes. For example, Cheah et al. (1998), Morton et al. (2001), Strawn et al. (2004), and Furnare et al. (2005) reported the coordination numbers (CNs) of the $\mathrm{Cu}$ (II) aqueous and adsorption complexes close to 4. Although a study by Pasquarello et al. (2001) suggested a five-fold coordination, the generally accepted $\mathrm{CN}$ of the first hydration shell of the aqueous 
$\mathrm{Cu}(\mathrm{II})$ is 6 (Ohtaki and Radnai, 1993). The knowledge gap between the EXAFS-determined CN and the well-established $\mathrm{CN}$ value of 6 primary hydration water molecules consistent with the $3 d^{9}$ electronic structure has not yet been satisfactorily filled.

Despite its excellence in precisely determining the local coordination environments around metal atoms, EXAFS has not been as effective in differentiating the axial oxygen atoms from equatorial oxygen atoms bound to $\mathrm{Cu}(\mathrm{II})$. In EXAFS data fitting, the axial oxygen has been either ignored or simply assumed to be present (Korshin et al., 1998). Moreover, the reported inter-atomic distance values for the axial oxygen atoms suffer from inconsistency, typically ranging from 2.1 to $2.6 \AA$ (Pasquarello et al., 2001). Since EXAFS has not been very successful in depicting an acceptable physical picture of the well-known $\mathrm{Cu}(\mathrm{II})$ aqua ion coordination, it is even more challenging to get reliable coordination information solely by EXAFS for $\mathrm{Cu}$ (II) ions in the interlayer of smectite minerals. To overcome these shortcomings, theoretical calculations and structural information independently obtained from electron paramagnetic resonance (EPR) and X-ray diffraction (XRD) are used in this work to guide the X-ray absorption spectroscopy (XAS) data analysis. Using this multi-technique approach, the XAS data of $\mathrm{Cu}(\mathrm{II})$ in the interlayer of montmorillonite (SAz-1) are rigorously analyzed for montmorillonite samples with different $\mathrm{Cu}$ (II) loading and degree of hydration. The samples are prepared under acidic $\mathrm{pH}$ condition to ensure that $\mathrm{Cu}(\mathrm{II})$ is bound to the interlayer rather than the edge surfaces of the mineral.

\section{Materials and Methods}

\subsection{Montmorillonite}


Cheto montmorillonite (SAz-1) from the Source Clays Repository (The Clay Minerals Society) was used for this study. It has the reported structural formula of $\left(\mathrm{Ca}_{0.39} \mathrm{Na}_{0.36} \mathrm{~K}_{0.02}\right)\left[\mathrm{Al}_{2.71} \mathrm{Mg}_{1.11} \mathrm{Fe}(\mathrm{III})_{0.12} \mathrm{Mn}_{0.01} \mathrm{Ti}_{0.02}\right]\left[\mathrm{Si}_{8.00}\right] \mathrm{O}_{20}(\mathrm{OH})_{4}$, with most of its structural charge arising from the octahedral substitution (Fripiat et al., 1979). It has a cation exchange capacity (CEC) of $120 \mathrm{meq} / 100 \mathrm{~g}$ and $\mathrm{N}_{2}$-BET surface area of $97.42 \pm 0.58 \mathrm{~m}^{2} / \mathrm{g}$ (Fripiat et al., 1979). For the $\mathrm{Cu}$ adsorption experiments, it was purified and prepared in its $\mathrm{Na}$ exchanged form following the procedure described elsewhere (Morton et al., 2001).

\subsection{Adsorption sample preparation}

The adsorption samples were prepared by reacting $0.2 \mathrm{~g}$ montmorillonite with $200 \mathrm{~mL}$ cupric nitrate solution at the acidic $\mathrm{pH}$ of 4.0 in $250 \mathrm{~mL}$ PPCopolymer centrifuge bottles (Nalgene) for 10 hours. After the adsorption, the suspension was centrifuged at 10,000 rpm (19,682 $\mathrm{g})$ for 20 minutes using a DuPont Sorvall RC-5B centrifuge and an RCA rotor. Then the supernatant solution was decanted and duplicate aliquots of $5 \mathrm{~mL}$ were collected for atomic absorption spectroscopy analysis for $\mathrm{Cu}$. The slurries were either kept wet or freeze-dried for further characterization by EXAFS, EPR, and XRD. All the solutions were prepared using distilled and deionized water (18.3 M $\Omega$-cm; Milli-Q water system).

A Cu-saturated montmorillonite $(\mathrm{Cu}-\mathrm{Mt})$ was prepared by reacting a $0.2 \mathrm{~g}$ dry montmorillonite with a $1,000 \mathrm{ppm}\left(1.57 \times 10^{-2} \mathrm{M}\right) \mathrm{Cu}(\mathrm{II})$ stock solution prepared using an ACS grade $\mathrm{Cu}\left(\mathrm{NO}_{3}\right)_{2} \cdot 4.5 \mathrm{H}_{2} \mathrm{O}$ (Aldrich Chemical). To saturate the mineral with $\mathrm{Cu}(\mathrm{II})$, the $200 \mathrm{~mL}$ of supernatant after the centrifugation was replaced by the same amount of the fresh $1000 \mathrm{ppm}$ $\mathrm{Cu}$ (II) solution and the $\mathrm{Cu}$ (II) loaded montmorillonite slurry was reacted with the $\mathrm{Cu}$ (II) solution again. This procedure was repeated for three times. The reaction $\mathrm{pH}$ was kept at 4.0 without 
added background electrolyte, to keep $\mathrm{Cu}(\mathrm{II})$ in the interlayer of the mineral rather than on the edge surfaces (Hyun et al., 2000). Montmorillonite with interlayer $\mathrm{Na}^{+}$partially exchanged by $\mathrm{Cu}^{2+}(\mathrm{NaCu}-\mathrm{Mt})$ was prepared by reacting the montmorillonite sample with a solution of $10^{-4} \mathrm{M}$ $\mathrm{Cu}(\mathrm{II})$ in a $0.01 \mathrm{M} \mathrm{NaNO}_{3}$ background electrolyte solution at $\mathrm{pH}$ 4.0. The final $\mathrm{Cu}$ (II) loading was $5.7 \mathrm{mg} \mathrm{Cu}(\mathrm{II}) / \mathrm{g}$ montmorillonite, corresponding to $15 \%$ of the reported CEC value. A 1,000 ppm cupric nitrate atomic absorption standard solution (Fisher Scientific) with added $\mathrm{NaNO}_{3}$ (Fisher Scientific) was used as a model compound for XAS measurements. The details of the $\mathrm{Cu}(\mathrm{II})$ samples prepared are summarized in Table 1.

Other samples and reference compounds include $\mathrm{Cu}$ (II) precipitates and $\mathrm{Cu}$ (II) loaded montmorillonite prepared at $\mathrm{pH} 10$. The $\mathrm{Cu}(\mathrm{II})$ precipitates were prepared by addition of $1 \mathrm{~N}$ $\mathrm{NaOH}$ and consequent abrupt $\mathrm{pH}$ rise of the cupric nitrate stock solution. The precipitates were made to have the same $\mathrm{Cu}(\mathrm{II})$ loading as $\mathrm{Cu}-\mathrm{Mt}$ by diluting the precipitates with dry $\mathrm{Na}$ montmorillonite. The basic $\mathrm{pH}$ sample was prepared to obtain $\mathrm{Cu}(\mathrm{II})$ sorbed to the edge surfaces of montmorillonite. The spectra of the different $\mathrm{Cu}(\mathrm{II})$ loaded motnmorillonite samples and model compounds were compared to figure out the structural characteristics of $\mathrm{Cu}$ (II) associated under different $\mathrm{pH}, \mathrm{Cu}(\mathrm{II})$ loading, and degree of hydration.

\subsection{EPR}

EPR is concerned with unpaired electrons and their chemical environments (Goodman and Hall, 1995). In the presence of external magnetic field, the degeneracy of the energies of electrons with different spin states is lifted, known as the Zeeman effect. When the applied electromagnetic radiation satisfies the following condition, a strong absorption or resonance occurs: 
$h v=\Delta E=g \mu_{B} B \quad[1]$

where $v$ is the frequency of the electromagnetic wave $(\mathrm{Hz}), h$ is Planck's constant, $\mu_{B}$ is the Bohr magneton $\left(9.2731 \times 10^{-24} \mathrm{~J} / \mathrm{T}\right)$, and $B$ is the applied magnetic field (Calas, 1988; Goodman and Hall, 1995). The proportionality constant $g$ is known as the spectroscopic splitting factor or simply $g$-factor. It is 2.0023 for a free electron, but varies when the electron is bound in a molecule or mineral, giving information regarding the chemical environment of the unpaired electron (Goodman and Hall, 1995; Pan and Nilges, 2014).

X-band ( 9.25 GHz) spectra of $\mathrm{Cu}(\mathrm{II})$-loaded montmorillonite samples (NaCu-Mt) of different hydration state prepared at different degree of freeze-drying were collected using a Bruker EMX spectrometer (Department of Chemistry, University of Michigan) at room temperature. Same amounts of $\mathrm{NaCu}-\mathrm{Mt}$ freeze-dried powder samples were put in quartz sample tubes (Wilmad Glass) and their EPR spectra were measured.

\subsection{XRD}

The 001-reflections of the $\mathrm{Cu}$-saturated montmorillonite $(\mathrm{Cu}-\mathrm{Mt})$ sample before and after the freeze-drying were recorded using a Rigaku Rotaflex X-ray diffractometer with a rotating anode X-ray source (Hanawalt Lab., University of Michigan). An oriented sample of wet Cu-Mt paste was prepared by loading the paste on a glass slide. No noticeable sign of dehydration was observed during the data collection. A dry bulk powder sample of freeze-dried Cu-Mt was prepared using an aluminum sample holder. A Ni-filtered, monochromated $\mathrm{Cu} K \alpha$ radiation generated at $40 \mathrm{kV} / 100 \mathrm{~mA}$ was used with a slit set of $0.5^{\circ}$ divergent, $0.5^{\circ}$ Soller, and $0.15 \mathrm{~mm}$ receiving slit. The data were collected for 1 second at each step of $0.02^{\circ} 2 \theta$ in the range of $2^{\circ}-$ $12^{\circ} 2 \theta$ in a step scan mode. 


\subsection{XAS data collection and analysis}

$\mathrm{Cu} K$-edge $(8979 \mathrm{eV}) \mathrm{X}$-ray absorption spectra were collected at the beam lines 4-3 and 11-2 of Stanford Synchrotron Radiation Lightsource (SSRL) using a Si(220) double-crystal monochromator at the beam energy of $3.0 \mathrm{GeV}$ and maximum beam current of $100 \mathrm{~mA}$. Wet paste and dry powder samples were loaded in Teflon sample cells with Kapton tape windows. Fluorescence spectra were collected using a Lytle detector or 13-element Ge array detector. To minimize the contribution from the higher order harmonics, the monochromator was detuned $50 \%$ at the highest energy position of the scan. The beam energy was calibrated using the simultaneously measured copper foil spectrum. To get improved signal to noise ratios, multiple scans (4-16) were collected.

The EXAFS can be formulated as

$$
\chi(k)=\frac{\Delta \mu}{\mu_{0}} \approx \frac{m}{4 \pi h^{2} k} \sum_{j} \frac{N_{j}}{R_{j}^{2}} t_{j}(2 k) e^{-2 R_{j} / \lambda} \sin \left[2 k R_{j}+2 \delta_{j}(k)\right] e^{-2 k^{2} \sigma_{j}^{2}}
$$

where $\mu_{0}$ is the absorption coefficient without EXAFS; $k$ is the electron wave vector $=2 \pi / \lambda_{e} ; N_{j}$ is the number of atoms in the $j$-th coordination sphere; $R_{j}$ is the average radial distance to the $j$-th atomic shell; $t_{j}(2 k)$ is the back-scattering amplitude for the $j$-th atoms; $\lambda$ is the mean free path of the electron; $\sigma_{j}^{2}$ is the Debye-Waller factor due to thermal vibrations or static disorder with $\sigma_{j}$ being the root mean square fluctuation (Sayers et al., 1971; Lytle et al., 1975; Stern, 1988).

Data analyses were performed using SixPack, EXAFSPAK, IFEFFIT, and FEFF8 code (Webb, 2002; George and Pickering, 2000; Newville, 2001; Ankudinov et al., 2002). Energy calibrated, acceptable scans were averaged and backgrounds were removed using linear fits below the absorption edge and spline fits above the edge. The background removal was 
performed using the IFEFFIT code. The spectra were then converted from the energy to the frequency space using the photo electron wave vector $k$. The normalized, $k^{3}$-weighted EXAFS spectra were Fourier-transformed for the $k$ range of 3-12 and used for FEFF fitting to get structural parameters. Phase shift and amplitude functions for each backscattering path were obtained from theoretical calculations using the FEFF8 code. To obtain the optimal structural parameters, including coordination numbers and interatomic distances, the Debye-Waller factor $\left(\sigma^{2}\right)$ and energy reference $E_{0}$ parameters were also floated during the fitting. The many-body factor $S_{0}^{2}$ was fixed at 0.9 to reduce the number of fitting parameters. To isolate the first shell contribution from the overall EXAFS pattern, the first shell peak on each Fourier transform function was back-transformed to get the filtered EXAFS for the first coordination shell. OPT of EXAFSPAK, the non-linear least-squares fitting subprogram, was used to obtain the best fit to the filtered EXAFS. The fitting results from the SixPack and EXAFSPAK were cross compared to each other to ensure the reliability of the results.

\subsection{Theoretical XAS calculations}

The XAS patterns of hypothetical, isolated $\mathrm{Cu}\left(\mathrm{O}_{\mathrm{eq}}\right)_{4}\left(\mathrm{O}_{\mathrm{ax}}\right)_{2}$ coordination complex models were calculated using the FEFF8 code to simulate the experimentally measured XAS spectra of interlayer $\mathrm{Cu}$ (II) under different $\mathrm{Cu}$ loading and hydration conditions. In the calculations, the bond length between $\mathrm{Cu}$ and $\mathrm{O}_{\mathrm{eq}}\left(R_{\mathrm{Cu}-\mathrm{Oeq}}\right)$ was fixed at $1.95 \AA$, while $R_{\mathrm{Cu}-\mathrm{Oax}}$ was allowed to vary between $2.10 \AA$ and $2.75 \AA$ to cover the reported range of the $\mathrm{Cu}-\mathrm{O}_{\mathrm{ax}}$ bond lengths. $R_{\mathrm{Cu}-\mathrm{Oax}}$ value of $1.95 \AA$ and infinity were also calculated for the regular octahedral coordination and square planar coordination as the two extreme cases of the axially elongated tetragonal coordination. Also calculated was the XAS spectrum of the tetrahedrally coordinated $\mathrm{CuO}_{4}$ with the bond 
length of $1.95 \AA$. In the XANES region, full multiple scattering calculations were preformed in the FEFF simulation of the $\mathrm{Cu}(\mathrm{II})$ spectra. The contributions of the backscattering from $\mathrm{O}_{\mathrm{eq}}$ and $\mathrm{O}_{\mathrm{ax}}$ to the overall calculated EXAFS spectra were isolated to evaluate the influence of changing coordination structures to the corresponding EXAFS patterns.

\section{Results and Discussion}

\subsection{XRD of the Cu-saturated montmorillonite}

The XRD results show that the fully hydrated and evacuated $\mathrm{Cu}-\mathrm{Mt}$ have the $d_{001}$-spacing of $19.5 \AA$ and $12.5 \AA$, respectively (Figure 1). Considering that montmorillonite with $\mathrm{Cu}$ as the interlayer cation has a further reduced basal spacing of $9.7 \AA$ upon heating at $150{ }^{\circ} \mathrm{C}$ (McBride and Mortland, 1974), the basal spacing values of this study confirm that the fully hydrated and evacuated montmorillonite accommodate an average of four water layers and one water layer in the interlayer between the two adjacent tetrahedral layers. At the acidic $\mathrm{pH}$ of 4 , the edge surfaces are positively charged and are not effective for $\mathrm{Cu}(\mathrm{II})$ adsorption due to the electrostatic repulsion. Instead, most of $\mathrm{Cu}(\mathrm{II})$ associated with montmorillonite have been shown to be in the interlayer at the similar acidic pH values (Hyun et al., 2000; Morton et al., 2001). Since the interlayers are saturated with $\mathrm{Cu}(\mathrm{II})$, the $d_{001}$-spacing reflects the coordination environment of $\mathrm{Cu}(\mathrm{II})$. The spacing of $19.5 \AA$ suggests that enough water molecules are present in the fully expanded, hydrated interlayer to allow interlayer $\mathrm{Cu}(\mathrm{II})$ to keep its six hydration water molecules, as in aqueous solutions. The reduced $d_{001}$ spacing of $12.5 \AA$ indicates that the confined space in the dehydrated interlayer is no longer favorable for the dynamic Jahn-Teller inversion or free tumbling. The interlayer is not large enough for interlayer $\mathrm{Cu}(\mathrm{II})$ to retain its axial oxygen 
ligands. Under this dehydrated condition, a square planar coordination is the most likely configuration, consistent with the one water layer thickness of the interlayer spacing. In this dehydrated interlayer, all the coordination structures except the square planar coordination would suffer from an excessive repulsion from the basal oxygen atoms since some of the $\mathrm{O}$ ligands would go beyond the basal $\mathrm{O}$ plane of the tetrahedral sheets along the $c$-axis. The one water layer thickness rules out the tetrahedral coordination of the interlayer $\mathrm{Cu}$ (II) (Morton et al., 2001), which requires two layers of water molecules. It can be deduced that the $\mathrm{Cu}(\mathrm{II})$ adsorption complexes should be regularly arranged such that their $z$-direction is parallel to the $c$-axis of the mineral. In conclusion, XRD suggests that a tetragonal coordination by six water molecules is possible for $\mathrm{Cu}(\mathrm{II})$ in the fully hydrated interlayer, whereas a square planar coordination by four oxygen ligands is the most likely coordination environment in the dehydrated interlayer.

\subsection{EPR of interlayer $\mathrm{Cu}(\mathrm{II})$}

At room temperature, $\mathrm{Cu}(\mathrm{II})$ in the freeze-dried $\mathrm{NaCu}-\mathrm{Mt}$ has two distinct EPR signals, viz., an isotropic signal with $g_{\text {iso }}=2.170$ and an anisotropic signal with $g_{\|}=2.338, g_{\perp}=2.054$, and $A_{\|}$ $=142 \mathrm{G}$ (Figure 2). On the other hand, only the isotropic signal was observed for $\mathrm{Cu}$ (II) in the fully hydrated montmorillonite (Hyun et al., 2000). The intensity of the two signals changes with degree of dehydration of the $\mathrm{Cu}$ loaded montmorillonite (Figure 3). With increasing degree of dehydration, the anisotropic signal intensified while the isotropic signal decreased simultaneously. Montmorillonite itself has EPR features between $\sim 3400$ and $\sim 3700 \mathrm{G}$ originating from structural $\mathrm{Fe}(\mathrm{III})$, but they neither affects or are affected by the $\mathrm{Cu}(\mathrm{II})$ adsorption or hydration state. 
The simultaneous observation of isotropic and anisotropic signals at room temperature suggests the mixed single and multiple layers of water in the interlayers. The isotropic signal was assigned as fully hydrated $\mathrm{Cu}(\mathrm{II})$ under the influence of the dynamic Jahn-Teller inversion with a free tumbling motion, leading to the average-out of the orientation-dependent contributions to the spin Hamiltonian, as in aqueous $\left[\mathrm{Cu}\left(\mathrm{H}_{2} \mathrm{O}\right)_{6}\right]^{2+}$ ions. The observation of the isotropic signal (Figure 2) indicates that $\mathrm{Cu}(\mathrm{II})$ is weakly held in the interlayer, keeping all its six water molecules of the first solvation shell. This conclusion is consistent with the electron spin-echo modulation (ESEM) study performed by Brown and Kevan (1988) that showed that $\mathrm{Cu}^{2+}$ has a hydration number of 6 in an essentially aqueous environment in the hydrated interlayer of montmorillonite.

The observation of the anisotropic signal suggests that $\mathrm{Cu}(\mathrm{II})$ in the dehydrated interlayer is in a motion-frozen state on the EPR timescale at room temperature. As the interlayer loses water, interlayer $\mathrm{Cu}$ stops the dynamic Jahn-Teller inversion or free tumbling motion, eventually reaching a motion-frozen status. The order of $g_{\|}>g_{\perp}>g_{\mathrm{e}}$ from the $\mathrm{Cu}(\mathrm{II})$ anisotropic signal indicates a $D_{4 h}$ symmetry or axially elongated octahedral coordination of $\mathrm{Cu}(\mathrm{II})$ due to the JahnTeller effect, ruling out the possibility of tetrahedral coordination (Figure 2 and 3) (Pilbrow, 1990). Moreover, the $g_{\|}$-value of the present study (2.338) is significantly lower than the previously reported values for the aqueous $\mathrm{Cu}(\mathrm{II})$, e.g., 2.409 as reported by Bassetti et al. (1979) and 2.4 by Matar et al. (1995), or the values reported for $\mathrm{Cu}(\mathrm{II})$ in the fully hydrated interlayer of montmorillonite, 2.41 for $\mathrm{Cu}(\mathrm{II})$ in a Mg-montmorillonite (Brown and Kevan, 1988) and 2.420 for that in a Na-montmorillonite measured at 77K (Hyun et al., 2000). Consistent with the reported empirical relation between $g_{\|}$-value and the stability constant (Motschi, 1984), this significant decrease of $g_{\|}$-value compared to that of six-fold coordinated $\mathrm{Cu}(\mathrm{II})$ suggests that 
interlayer $\mathrm{Cu}(\mathrm{II})$ has lost part of its primary hydration sheath in the dehydrated interlayer. The most feasible explanation is that $\mathrm{Cu}(\mathrm{II})$ has lost the axial oxygen atoms from its first hydration shell as a result of dehydration. Loss of the longer, weaker $\mathrm{Cu}-\mathrm{O}_{\mathrm{ax}}$ bonds would result in the observed loss of fast water exchange with the neighboring water molecules. Therefore, the anisotropic signal observed in the dehydrated interlayer is assigned as $\mathrm{Cu}(\mathrm{II})$ in a square planar geometry. Consequently, the intensity change of the two signals (Figure 3) indicates the transition of $\mathrm{Cu}(\mathrm{II})$ coordination from a tetragonal to a square planar coordination with dehydration. This interpretation is also consistent with the EPR results of $\mathrm{Cu}^{2+}$ on various expandable layer silicates reported by McBride (1982; 1986).

\subsection{XANES spectra of interlayer $\mathrm{Cu}(\mathrm{II})$}

The XANES spectra of interlayer $\mathrm{Cu}$ (II) in montmorillonite prepared under different $\mathrm{Cu}$ (II) loading and hydration conditions are plotted in Figure 4, along with those of $\mathrm{Cu}$ (II) model compounds. Of all the samples and model compounds, the fully hydrated $\mathrm{Cu}$ (II) saturated montmorillonite (Cu-Mt-w) is the only one with a singlet $1^{\text {st }}$ derivative XANES spectrum. All other samples and model compounds have doublet $1^{\text {st }}$ derivative XANES spectra. The position and relative intensity of the two peaks and the splitting between the two peaks change depending on $\mathrm{Cu}$ loading and degree of hydration. For example, $\mathrm{Cu}(\mathrm{II})$ aqua ion has the double peaks at $8988.25 \mathrm{eV}$ and $8992.25 \mathrm{eV}$, with the pre-edge feature at $8975.75 \mathrm{eV}$. The splitting between the two peaks of the doublet is $4 \mathrm{eV}$. Cupric nitrate solid has the doublet at $8988.50 \mathrm{eV}$ and $8992.00 \mathrm{eV}$ with the splitting of $3.5 \mathrm{eV}$. Cu-saturated, dehydrated montmorillonite (Cu-Mt-d) has the doublet at $8984.99 \mathrm{eV}$ and $8990.62 \mathrm{eV}$ with the splitting of $5.63 \mathrm{eV}$. 
Figure 5 shows the simulation results of the $1^{\text {st }}$ derivative XANES spectra for the different $\mathrm{Cu}$ (II) coordination, namely, the regular octahedral, axially elongated tetragonal, and square planar coordination. The regular octahedral has a singlet feature, whereas tetragonal and square planar have doublet features. Figure 6 shows the simulation results of the $1^{\text {st }}$ derivative XANES spectra for the tetragonal coordination as a function of $\mathrm{Cu}-\mathrm{O}_{\mathrm{ax}}$ distance with the fixed $\mathrm{Cu}-\mathrm{O}_{\mathrm{eq}}$ distance at $1.95 \AA$. The results show that the splitting between the two peaks increases with the increasing $\mathrm{Cu}-\mathrm{O}_{\mathrm{ax}}$ distance.

\subsection{EXAFS spectra of interlayer $\mathrm{Cu}(\mathrm{II})$}

Two approaches were used in analyzing the EXAFS spectra of $\mathrm{Cu}(\mathrm{II})$ in aqueous solution and in the hydrated interlayer: a one-path $(\mathrm{Cu}-\mathrm{O})$ fitting with a variable coordination number $(\mathrm{CN})$ and two-path $\left(\mathrm{Cu}-\mathrm{O}_{\mathrm{eq}} \& \mathrm{Cu}-\mathrm{O}_{\mathrm{ax}}\right)$ fitting with fixed coordination numbers of 4 and 2 for the $\mathrm{O}_{\mathrm{eq}}$ and $\mathrm{O}_{\mathrm{ax}}$, respectively. The 1-path fitting was not based on any structural model. The first shell EXAFS parameters were obtained using one $\mathrm{Cu}-\mathrm{O}$ path while varying both the $\mathrm{CN}$ and the corresponding Debye-Waller factor $\left(\sigma^{2}\right)$. This approach gives the fewest number of fitting parameters. The 2-path fitting was based on the $4+2$ coordination model where $\mathrm{Cu}$ (II) has the

first coordination shell composed of four closer equatorial oxygen $\left(\mathrm{O}_{\mathrm{eq}}\right)$ atoms and two farther axial oxygen $\left(\mathrm{O}_{\mathrm{ax}}\right)$ atoms. This approach is guided by the structural information obtained from XRD, EPR, and XANES. The regular octahedral and square planar coordination can be incorporated into the $4+2$ structure model as the two extreme cases. The goodness of the fit value ( $R$-factor from the SixPack or $F$-factor from the OPT output data) was used as a parameter in comparing the closeness of the fitting results to the measured data: the lower is the value, the 
better is the fitting. The fitting results from the filtered and back-transformed first shell EXAFS were checked using the unfiltered, overall spectra.

The results of the theoretical calculations of the EXAFS spectra of the hypothetical $\mathrm{Cu}$ (II) model $\mathrm{Cu}\left(\mathrm{O}_{\mathrm{eq}}\right)_{4}\left(\mathrm{O}_{\mathrm{ax}}\right)_{2}$ with different $\mathrm{Cu}-\mathrm{O}_{\mathrm{ax}}$ bond lengths in a tetragonally elongated octahedral coordination are given in Figure 7. The contributions from $\mathrm{O}_{\mathrm{eq}}$ and $\mathrm{O}_{\mathrm{ax}}$ are not well resolved and no distinct beat pattern is observed in the overall EXAFS pattern when the two contributions are combined (Figure 7). The differences in the oscillation frequency, amplitude envelope, and amplitude damping pattern between the overall EXAFS and those from $\mathrm{O}_{\mathrm{eq}}$ are not distinct. In general, the relative contributions from $\mathrm{O}_{\mathrm{ax}}$ to the overall EXAFS are minor and dependent on the axial bond $\left(\mathrm{Cu}-\mathrm{O}_{\mathrm{ax}}\right)$ length. The two contributions are not well resolved and a distinct, resolvable, and separable contribution from $\mathrm{O}_{\mathrm{ax}}$ is not observed (Figure 7). These example calculations show that special caution is required to properly interpret the experimental EXAFS data for the aqueous and interlayer species of $\mathrm{Cu}(\mathrm{II})$. The destructive interference between the backscattering from $\mathrm{O}_{\mathrm{eq}}$ and $\mathrm{O}_{\mathrm{ax}}$ leads to the underestimation of the $\mathrm{CN}$, close to 4 rather than 6 , if analyzed using the one-path fitting without a reliable structural model. These theoretical calculations demonstrate the reason why $\mathrm{Cu}-\mathrm{O}_{\mathrm{ax}}$ bond has often been either ignored in the EXAFS fitting or reported with inconsistent bond length values in the previous studies. This result shows that an alternative EXAFS fitting strategy is needed that is guided by reliable structural information from independent sources for a better analysis of $\mathrm{Cu}(\mathrm{II})$ EXAFS data.

\subsubsection{EXAFS of $\mathrm{Cu}$ aqua ion}

The best 1-path fitting for the $\mathrm{Cu}(\mathrm{II})$ aqua ion while floating both the $\mathrm{CN}$ and $\sigma^{2}$ gives the first hydration shell of 3.3 nearest oxygen atoms located $1.964 \AA$ from the central copper atom with 
$\sigma^{2}$ of 0.0039 (Table 2). The fitting underestimates the $\mathrm{CN}$, compared to the generally accepted value of six solvation water molecules for $\mathrm{Cu}(\mathrm{II})$ in aqueous solutions (Ohtaki and Radnai, 1993; Richens, 1997). As the theoretical calculations in the preceding section show, the destructive contribution from the axial oxygen atoms is responsible for this significant underestimation of the $\mathrm{CN}$. This deviation of the $\mathrm{CN}$ from the known value again demonstrates the need for a reliable fitting strategy to extract physically meaningful information from the experimental $\mathrm{Cu}$ EXAFS data, since the fitting without known structural information can mislead to the conclusion of 'tetrahedral' coordination of aqueous $\mathrm{Cu}(\mathrm{II})$ ions with the contribution from the $\mathrm{Cu}-\mathrm{O}_{\mathrm{ax}}$ path being neglected (Morton et al., 2001).

The 2-path fitting using the 4+2 structural model gives the best fit of four $\mathrm{O}_{\text {eq }}$ atoms at 1.969 $\AA$ with $\sigma^{2}$ of 0.0047 and two $\mathrm{O}_{\mathrm{ax}}$ atoms at $2.138 \AA$ with $\sigma^{2}$ of 0.0149 (Table 2). These EXAFS parameters are consistent with the generally accepted local coordination structure of the aqueous $\mathrm{Cu}(\mathrm{II})$ ions. The two $\mathrm{Cu}-\mathrm{O}$ distances are in the reported range previously determined using XRD or ND (Pasquarello et al., 2001). The higher $\sigma^{2}$ value for the $\mathrm{Cu}-\mathrm{O}_{\mathrm{ax}}$ bond compared to that for $\mathrm{Cu}-\mathrm{O}_{\mathrm{eq}}$ is consistent with the physical picture of the loosely bound axial oxygen atoms under fast exchange with neighboring water molecules. The addition of the $\mathrm{O}_{\mathrm{ax}}$ neighbors also improves the goodness of the fit (the $R$-factor), from 0.0420 for the 1-path fitting to 0.0372 for the 2 -path fitting, even though the highly inter-dependent $\mathrm{CN}$ and $\sigma^{2}$ are both floated in the 1-path fitting significantly improving the goodness of the fitting. This comparison shows that the application of the 4+2 model with fixed CNs gives more physically reliable depiction of the coordination environment of the $\mathrm{Cu}(\mathrm{II})$ aqua ions, as well as slightly better fitting result.

Since the timescale of the EXAFS events is much shorter than the inter-conversion of the axis of elongation of the aqueous $\mathrm{Cu}(\mathrm{II})$ under the Jahn-Teller effect, EXAFS is expected to enable 
the determination of a weighted average of the interatomic distances (Persson et al, 2002). However, the atomic shell-based EXAFS analysis using Eq. [2] was not efficient in exactly depicting the coordination structure including the orientation of the oxygen ligands in the shell, since the oxygen backscatterers are not evenly distributed in the shell in the tetragonal or square planar complexes. Moreover, when the two oxygen atomic 'shells' are too close to be resolved, which is the case for the $\mathrm{Cu}(\mathrm{II})$ aqua ion, the EXAFS analysis can lead to unreliable structural information.

\subsubsection{EXAFS of interlayer $\mathrm{Cu}$}

The EPR and XRD results consistently suggest the tetragonal and square planar coordination for $\mathrm{Cu}(\mathrm{II})$ in the fully hydrated and dehydrated interlayer, respectively. The EXAFS data of $\mathrm{Cu}$ (II) in the hydrated interlayer are analyzed following the same procedure used for the $\mathrm{Cu}$ (II) aqua ion, i.e., 2-path fitting using the $4+2$ structural model and 1-path fitting without any structural model. The fitting results are summarized in Table 2 .

The 2-path model with fixed CNs gives a better fit with $4 \mathrm{O}_{\mathrm{eq}}$ atoms at $1.900 \AA$ and $2 \mathrm{O}_{\mathrm{ax}}$ atoms at $1.955 \AA(R=0.0138)$ for $\mathrm{Cu}(\mathrm{II})$ in the wet $\mathrm{Cu}$-saturated montmorillonite $(\mathrm{Cu}-\mathrm{Mt}-\mathrm{w})$, compared to the 1-path fit with $3.2 \mathrm{O}$ at $1.959 \AA$ ( $R=0.0189$; Table 2 and Figure $8 \mathrm{a}$ and b). These CNs and bonding distances are supportive of the coordination close to the regular octahedral structure. The $\mathrm{Cu}-\mathrm{O}_{\text {eq }}$ bond length is slightly shorter than the commonly reported $\sim 1.95 \AA$, but longer than the short $\mathrm{Cu}-\mathrm{O}$ bond length of $1.89843 \AA$ for malachite (Zigan et al., 1977) and $1.87947 \AA$ for azurite (Wyckoff, 1978), the cupric hydroxycarbonate minerals.

XRD shows that the dehydrated interlayer has only one layer of water molecules, suggesting the square planar coordination of interlayer $\mathrm{Cu}(\mathrm{II})$. The 1-path fitting gives the fitting result with 
the structural parameters of $4 \mathrm{O}$ at $1.941 \AA$ with $\sigma^{2}$ of $0.0059(R$-factor $=0.0430)$, while the 2 path fitting did not converge to a set of reasonable structural parameters. The addition of a multiple scattering path of $\mathrm{O}_{\mathrm{eq} 1}-\mathrm{Cu}-\mathrm{O}_{\mathrm{eq} 3}$ at $3.92 \AA$ improved the goodness of the fit to the measured EXAFS. Although the presence of the multiple scattering path is suggestive of square planar coordination, while discarding the possibility of tetrahedral coordination, the Fourier transform magnitude of the multiple scattering contribution in the radial structure function was not significant enough and was not included in the result presented in Table 2.

Upon wetting the dehydrated $\mathrm{Cu}-\mathrm{Mt}$ by adding a small amount of water, interlayer $\mathrm{Cu}(\mathrm{II})$ immediately recovers the EXAFS parameters same as the one before dehydration or $\mathrm{Cu}-\mathrm{Mt}-\mathrm{w}$. This observation suggests that $\mathrm{Cu}$ coordination reversibly and readily interchangeable between octahedral and square planar coordination depending on the availability of water molecules and the hydration state of the interlayer.

The EXAFS parameters of the first coordination shell of the interlayer $\mathrm{Cu}(\mathrm{II})$ sorbed on the fully hydrated montmorillonite with the surface $\mathrm{Cu}$ loading of $5.7 \mathrm{mg} / \mathrm{g}$ montmorillonite $(\mathrm{NaCu}-$ Mt-w) are similar to those of the $\mathrm{Cu}(\mathrm{II})$ aqua ions (Table 2 and Figure $8 \mathrm{c}$ and d). The 2-path fitting indicates four $\mathrm{O}_{\text {eq }}$ neighbors at $1.976 \AA$ with $\sigma^{2}$ of 0.0050 and two $\mathrm{O}_{\mathrm{ax}}$ neighbors at 2.277 $\AA$ with $\sigma^{2}$ of 0.0148 . For the 1-path fitting the first coordination shell is composed of 3.6 oxygen atoms at $1.961 \AA$ with $\sigma^{2}$ of 0.0046 . The $R$-factor is 0.0161 for the 2-path fitting, better than 0.0278 for the 1 -path fitting.

As EPR shows, interlayer $\mathrm{Cu}(\mathrm{II})$ ions cannot retain the axial oxygen atoms in the dehydrated interlayer. The 1-path fitting gives $4 \mathrm{O}$ at 1.942 with $\sigma^{2}$ of 0.0057 and $R$-factor of 0.0421 for the dry $\mathrm{NaCu}-\mathrm{Mt}$ (Table 2). In the present study, the estimated error for the EXAFS determination of the interatomic distance for the equatorial oxygen atoms was $\pm 0.006 \AA$ for $\mathrm{NaCu}-\mathrm{Mt}$. To be 
conservative, using the error value $( \pm 0.01 \AA)$ reported in literature (Korshin et al., 1998; Morton et al., 2001) for the first shell interatomic distance for $\mathrm{Cu}(\mathrm{II})$ complexes, the $\mathrm{Cu}-\mathrm{O}_{\text {eq }}$ bond length in the dry interlayer $(1.942 \AA)$ is shorter than that of tetragonal coordination in the wet $\mathrm{NaCu}-\mathrm{Mt}$

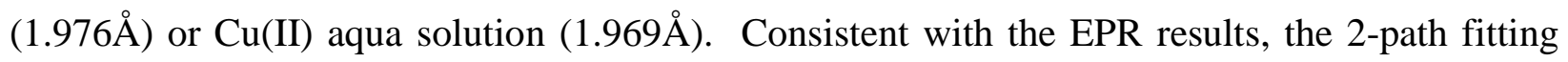
does not converge to a physically acceptable set of structural parameters. Nor does the addition of the extra $\mathrm{Cu}-\mathrm{O}_{\mathrm{ax}}$ path improve the fitting.

\subsection{Model for interlayer $\mathrm{Cu}(\mathrm{II})$ coordination}

Putting together the information obtained from EPR, XRD, XANES, EXAFS, and theoretical calculations, a structural model is proposed for the coordination environment of interlayer $\mathrm{Cu}(\mathrm{II})$ in the $\mathrm{Cu}$-saturated/unsaturated and hydrated/dehydrated montmorillonite (Table 3). In the fully hydrated, $\mathrm{Cu}$-saturated montmorillonite $(\mathrm{Cu}-\mathrm{Mt}-\mathrm{w}), \mathrm{Cu}(\mathrm{II})$ is bound to six water molecules in a close to regular octahedral coordination. This configuration is supported by the singlet $1^{\text {st }}$ derivative XANES spectrum and the corresponding FEFF simulation. In the fully hydrated, $\mathrm{Cu}$-unsaturated montmorillonite $(\mathrm{NaCu}-\mathrm{Mt}-\mathrm{w}), \mathrm{Cu}(\mathrm{II})$ is coordinated by four closer equatorial oxygen atoms and two farther axial oxygen atoms. In this configuration, rapid water exchange at the axial oxygen atoms and change of the axis of elongation occur, as supported by EPR. Interlayer $\mathrm{Cu}(\mathrm{II})$ is bonded to four equatorial oxygen atoms in the dehydrated interlayer both in a $\mathrm{Cu}$-saturated and unsaturated montmorillonite. Now $\mathrm{Cu}$ (II) lacks the fast water exchange or dynamic Jahn-Teller inversion, and a stronger interaction with the clay layers is expected.

The saturated interlayer has only $\mathrm{Cu}^{2+}$ ions and water molecules in the interlayer. The interlayer space has a difference from the aqueous solution in that it lacks the counter ions such 
as $\mathrm{Cl}^{-}, \mathrm{NO}_{3}^{-}, \mathrm{ClO}_{4}^{-}$, and $\mathrm{SO}_{4}{ }^{2-}$, the common components in the aqueous solution of $\mathrm{Cu}(\mathrm{II})$ salts. The Cu-saturated interlayer has a relatively homogeneous coordination environment compared to the unsaturated interlayer. The unsaturated interlayer has $\mathrm{Na}^{+}$as well as $\mathrm{Cu}^{2+}$. The interlayer property is largely determined by the interlayer $\mathrm{Na}^{+}$, affecting the $\mathrm{Cu}(\mathrm{II})$ coordination as well..

The swelling phenomena of montmorillonite have long been recognized. The reversible expansion and contraction of the interlayer correspond with the conversion of copper coordination between the tetragonal and square planar configuration, suggesting that square planar $\mathrm{Cu}(\mathrm{II})$ can easily convert to the axially elongated tetragonal $\mathrm{Cu}(\mathrm{II})$ when sufficient hydration water is available. The reversible conversion of coordination is evidence by XRD, EPR, and XAS between hydrated and dehydrated montmorillonite samples as discussed in the previous (3.4.) section. Similar reversible conversion between the two configurations was also reported for $\mathrm{Cu}$ (II) sorbed on kaolinite (Hyun et al., 2005). The readily reversible rehydration of the interlayer rules out the likelihood of conversion to the tetrahedral configuration.

This study first suggests the regular octahedral coordination of $\mathrm{Cu}(\mathrm{II})$ in the interlayer of montmorillonite. It also reports the exchangeability between octahedral, tetragonal, and square planar coordination of interlayer $\mathrm{Cu}(\mathrm{II})$ depending on the physical conditions that are readily adjustable. The findings of this study are expected to have practical implications such as in catalysts and medical applications. The analytical approaches used in this study would be expanded to probe the chemical environments of the interlayer sites in other expandable clay minerals. Further development can also be made by combing the analytical approaches with coordination theories and quantum mechanical calculations on $\mathrm{Cu}(\mathrm{II})$ coordination as a function of layer charge in a series of expandable phyllosilicate minerals. 


\section{Conclusions}

Complementary EPR, XRD, and XAS provided independent structural information on the $\mathrm{Cu}(\mathrm{II})$ coordination, allowing a more definitive assignment of the coordination of $\mathrm{Cu}(\mathrm{II})$ in the interlayer of montmorillonite. Interlayer $\mathrm{Cu}(\mathrm{II})$ in the $\mathrm{Cu}$-saturated, fully hydrated montmorillonite has a quasi-regular octahedral coordination. Interlayer $\mathrm{Cu}(\mathrm{II})$ in unsaturated, fully hydrated montmorillonite has an axially elongated tetragonal coordination. Dehydrated montmorillonite has interlayer $\mathrm{Cu}(\mathrm{II})$ in a square planar coordination. This study newly suggests the quasi-regular octahedral coordination and shows that the different coordination structures are interchangeable depending on the hydration state and $\mathrm{Cu}(\mathrm{II})$ loading conditions.

Theoretical EXAFS calculations show that the destructive interference caused by the slight difference in the bond distance between $\mathrm{Cu}-\mathrm{O}_{\mathrm{eq}}$ and $\mathrm{Cu}-\mathrm{O}_{\mathrm{ax}}$ can confound the analysis of the experimental $\mathrm{Cu}$ (II) EXAFS data. EXAFS analysis based on the $4+2$ structural model was able to provide physically acceptable coordination structures around interlayer $\mathrm{Cu}(\mathrm{II})$. Since the 1 path fitting could often provide fitting results comparable to the 2-path fitting in terms of the goodness of the fit, it might be considered as the better approach in terms of simplicity. However, as this study shows it is not physically acceptable when viewed in the context of the complementary EPR and XRD results. This work shows that EXAFS should be applied with caution to the study of transition metal coordination on clay minerals, particularly for metals in which multiple first shell paths are the norm in aqueous environments such as with aqua $\mathrm{Cu}(\mathrm{II})$.

\footnotetext{
Acknowledgments. The authors thank Dr. H.Y. Jeong (Pusan Nation University, Pusan, Korea) and Dr. C.-C. Chen (the University of Texas-Austin) for their help in EXAFS data collection.
} 
Part of this research was funded by U.S. DOE-Environmental Management Science Program (Grant\# DE-FG07-97ER45652) and KIGAM's Basic R\&D program (GP2014-020). Portion of this research were carried out at the Stanford Synchrotron Radiation Lightsource, A Directorate of SLAC National AcceleratorLaboratory and an Office of Science User Facility operated for the U.S. Department of Energy Office of Science by Stanford University. The SSRL Structural Molecular Biology Program is supported by the DOE Office of Biological and Environmental Research, and by the National Institute of Health, National Center for Research Resources, Biomedical Technology Program (P41RR001209). 


\section{References}

R.A. Alvarez-Puebla, C. Aisa, J. Blasco, J.C. Echeverria, B. Mosquera, J.J. Garrido (2004). Copper heterogeneous nucleation on a palygorskitic clay: an XRD, EXAFS, and molecular modeling study. Applied Clay Science 25, 103-110.

A. Ankudinov, B. Ravel, J.J. Rehr, FEFF8, The FEFF Project, Department of Physics, University of Washington, 2002.

V. Bassetti, L. Burlamacchi, G. Martini (1979). Use of paramagnetic probes for the study of liquid adsorbed on porous supports. Journal of the American Chemical Society 101, 54715477.

K.G. Bhattacharyya, S.S. Gupta (2006). Adsorptive accumulation of $\mathrm{Cd}(\mathrm{II}), \mathrm{Co}(\mathrm{II}), \mathrm{Cu}(\mathrm{II}), \mathrm{Pb}$ (II), and $\mathrm{Ni}(\mathrm{II})$ from water on montmorillonite: Influence of acid activation. Journal of Colloid and Interface Science 310, 411-424.

M.F. Brigatti, S. Colonna, D. Malferrari, L. Medici (2004). Characterization of Cu-complexes in smectite with different layer charge location: Chemical, thermal, and EXAFS studies. Geochim. Cosmochim. Acta. 68, 781-788.

D.R. Brown, L. Kevan (1988). Aqueous coordination and location of exchangeable copper(2+) cations in montmorillonite clay studied by electron spin resonance and electron spin echo modulation. Journal of the American Chemical Society 110, 2743-2748.

G. Calas (1988). Electron paramagnetic resonance. In: Spectroscopic methods in mineralogy and geology. (Editor: F.C. Hawthorne). Reviews in Mineralogy 18, Mineralogical Society of America. 
S.-F. Cheah, G.E. Brown Jr., G.A. Parks (1998) XAFS spectroscopy study of Cu(II) sorption on amorphous $\mathrm{SiO}_{2}$ and $\gamma-\mathrm{Al}_{2} \mathrm{O}_{3}$ : Effect of substrate and time on sorption complexes. Journal of Colloid and Interface Science 208, 110-128.

A.A. El-Bayaa, N.A. Badawy, E.A. Alkhalik (2009). Effect of ionic strength on the adsorption of copper and chromium ions by vermiculite pure clay mineral. Journal of Hazardous Materials 170, 1204-1209.

C.A. Flemming, J.T. Trevors (1989). Copper toxicity and chemistry in the environment: a review. Water, Air, and Soil Pollution 44, 143-158.

J.J. Fripiat, H. Van Olphen, Data Handbook for Clay Materials and Other Non-metallic Minerals, Pergamon Press, New York, 1979.

L.J. Furnare, A. Vailionis, D.G. Strawn (2005) Molecular-level investigation into copper complexes on vermiculite: Effect of reduction of structural iron on copper complexation. Journal of Colloid Interface Science 289, 1-13.

G.N. George, I.J. Pickering, EXAFSPAK, A Suite of Computer Programs for Analysis of X-ray Absorption Spectra, Stanford Synchrotron Radiation Laboratory, Stanford Linear Accelerator Center, Menlo Park, 2000.

B.A. Goodman, P.L. Hall (1995). Electron paramagnetic resonance spectroscopy. In: Clay mineralogy: Spectroscopic and chemical determinative methods (Editor: M.J. Wilson). Chapman \& Hall, London.

S.P. Hyun, Y.H. Cho, P.S. Hahn (2005) An electron paramagnetic resonance study of $\mathrm{Cu}(\mathrm{II})$ sorbed on kaolinite. Applied Clay Science 30, 69-78. 
S.P. Hyun, Y.H. Cho, S.J. Kim, P.S. Hahn (2000). $\mathrm{Cu}(\mathrm{II})$ sorption mechanism on montmorillonite: An electron paramagnetic resonance study. Journal of Colloid and Interface Science 222, 254-261.

G.V. Korshin, A.I. Frenkel, E.A. Stern (1998) EXAFS study of the inner shell structure in copper(II) complexes with humic substances. Environmental Science and Technology 32, 2699-2705.

F.W. Lytle, D.E. Sayers, E.A. Stern (1975) Extended X-ray absorption fine structure technique. II. Experimental practice and selected results. Physical Review B11, 4825-4835.

K. Matar, D. Zhao, D. Goldfarb, W. Azelee, W. Daniel, P.G. Harrison (1995) Characterization of $\mathrm{Cu}$ (II) sites in $\mathrm{Cu} / \mathrm{SnO}_{2}$ catalysts by electron spin echo envelope modulation spectroscopy. Journal of Physical Chemistry 99, 9966-9973.

M.B. McBride (1982) Hydrolysis and dehydration reactions of exchangeable $\mathrm{Cu}^{2+}$ on hectorite. Clays and Clay Minerals 30, 200-206.

M.B. McBride, in: J.A. Davis, K.F. Hayes (Eds.), Geochemical Processes at Mineral Surfaces, American Chemical Society, Washington, DC, 1986, pp.362-388.

M.B. McBride, M.M. Mortland (1974) Copper(II) interactions with montmorillonite: Evidence from physical methods. Soil Science Society of America Journal 38, 408-415.

J.D. Morton, J.D. Semrau, K.F. Hayes (2001) An X-ray absorption spectroscopy study of the structure and reversibility of copper adsorbed to montmorillonite clay. Geochimica et Cosmochimica Acta 65, 2709-2722.

H. Motschi (1984) Correlation of EPR-parameters with thermodynamic stability constants for copper(II) complexes $\mathrm{Cu}$ (II)-EPR as a probe for the surface complexation at the water/oxide interface. Colloids Surfaces 9, 333-347. 
M. Newville, The IFEFFIT Tutorial, Consortium for Advanced Radiation Sources, University of Chicago, Chicago, 2001.

H. Ohtaki, T. Radnai (1993) Structure and dynamics of hydrated ions. Chemical Review 93, 1157-1204.

Y. Pan, M.J. Nilges (2014). Electron paramagnetic resonance spectroscopy: Basic principles, experimental techniques and applications to earth and planetary sciences. In: Spectroscopic methods in mineralogy and materials sciences. (Editors: G.S. Henderson, D.R. Neuville, R.T. Downs). Reviews in Mineralogy and Geochemistry 78. Mineralogical Society of America, Geochemical Society.

A. Pasquarello, I. Petri, P.S. Salmon, O. Parisel, R. Car, E. Toth, D.H. Powell, H.E. Fischer, L. Helm, A.E. Merbach (2001) First solvation shell of the $\mathrm{Cu}(\mathrm{II})$ aqua ion: Evidence for fivefold coordination. Science 291, 856-859.

I. Persson, P. Persson, M. Sandstrom, A.-S. Ullstrom (2002) Structure of Jahn-Teller distorted solvated copper(II) ions in solution, and in solids with apparently regular octahedral coordination geometry. Journal of the Chemical Society, Dalton Transactions, 1256-1265.

J.R. Pilbrow, Transition Ion Electron Paramagnetic Resonance, Oxford University Press, New York, 1990.

D.T. Richens, The Chemistry of Aqua Ions: Synthesis, Structure and Reactivity, John Wiley \& Sons Ltd., Chichester, 1997.

B.M. Ryan, J.K. Kirby, F. Degryse, K. Scheiderich, M.J. McLaughlin (2014). Copper isotope fractionation during equilibrium with natural and synthetic ligands. Environ. Sci. Technol. 48, 8620-8626. 
D.E. Sayers, F.W. Lytle, E.A. Stern (1971) New technique for investigating noncrystalline structures: Fourier analysis of the extended X-ray-absorption fine structure. Physical Review Letters 27, 1204-1207.

M.L. Schlegel, A. Manceau (2013). Binding mechanism of $\mathrm{Cu}(\mathrm{II})$ at the clay-water interface by powder and polarized EXAFS spectroscopy. Geochim. Cosmochim. Acta 113, 113-124.

D.G. Strawn, N.E. Palmer, L.J. Furnare, C. Goodell, J.E. Amonenette, R. Kukkadapu (2004) Copper sorption mechanisms on smectites. Clays and Clay Minerals 52, 321-333.

E.A. Stern, in: D.C. Koningsberger, R. Prins (Eds.), X-ray Absorption. Principles, Applications, Techniques of EXAFS, SEXAFS, and XANES, John Wiley \& Sons, Inc., New York, 1988.

S. Veli, B. Alyuz (2007). Adsorption of copper and zinc from aqueous solutions by using natural clay. Journal of Hazardous Materials 149, 226-233.

S.M. Webb. Sam's Interface for XAS Package (SixPACK); Stanford Synchrotron Radiation Laboratory: Menlo Park, CA, 2002.

Wyckoff, R.W.G. Crystal Structures. John Wiley \& Sons. New York, 1978.

I. Yruela (2009). Copper in plants: acquisition, transport and interactions. Functional Plant Biology 36, 409-430.

Zigan, F., Joswig, W., Schuster, H.D., and Mason, S.A. (1977) Verfeinerung der struktur von malachi, $\mathrm{Cu}_{2}(\mathrm{OH})_{2} \mathrm{CO}_{3}$, durch neutronenbeugung. Zeitschrift fur Kristallographie. 145, 412. 
Table 1. Samples used in the present work.

\begin{tabular}{ccccc}
\hline Sample & $\mathbf{p H}$ & $\begin{array}{c}{[\mathbf{N a}(\mathbf{I})]_{\text {tot }}} \\
(\mathbf{m o l} / \mathbf{L})\end{array}$ & $\begin{array}{c}{[\mathbf{C u}(\mathbf{I I})]_{\text {tot }}} \\
(\mathbf{m o l} / \mathbf{L})\end{array}$ & $\begin{array}{c}\text { Cu loading } \\
\mathbf{m g ~ C u} / \mathbf{g} \text { Mont. } \\
(\% \mathbf{C E C})\end{array}$ \\
\hline Cu solution & 4.0 & 0 & $1.57 \times 10^{-2}$ & - \\
\hline NaCu-Mt & 4.0 & 0.01 & $10^{-4}$ & 5.7 \\
& & & & $(15 \%)$ \\
\hline Cu-Mt & 4.0 & 0 & $1.57 \times 10^{-2}$ & 38 \\
& & & three times & $(100 \%)$ \\
\hline
\end{tabular}


Table 2. Structural parameters of $\mathrm{Cu}$ aqua ion and interlayer $\mathrm{Cu}$ extracted from EXAFS analysis (C.N.: coordination number, $R$ : interatomic distance, $\sigma^{2}$ : Debye-Waller factor, *: fixed during fitting, $R$-factor: goodness of the fit). Numbers in bold are the selected final fitting result. Lower $R$-factor value indicates a better fitting to the measured data.

\begin{tabular}{|c|c|c|c|c|c|c|}
\hline Samples & & & C.N. & $R(\AA)$ & $\sigma^{2}$ & $R$ - factor \\
\hline \multirow{3}{*}{ Aqueous $\mathrm{Cu}$ (II) } & 1-path & $\mathrm{Cu}-\mathrm{O}$ & 3.3 & 1.964 & 0.0039 & 0.0420 \\
\hline & \multirow{2}{*}{ 2-path } & $\mathrm{Cu}-\mathrm{O}_{\mathrm{eq}}$ & $4 *$ & 1.969 & 0.0047 & \multirow{2}{*}{0.0372} \\
\hline & & $\mathrm{Cu}-\mathrm{O}_{\mathrm{ax}}$ & $2 *$ & 2.138 & 0.0149 & \\
\hline \multirow{3}{*}{ Cu-Mt-w } & 1-path & $\mathrm{Cu}-\mathrm{O}$ & 3.2 & 1.959 & 0.0038 & 0.0189 \\
\hline & \multirow{2}{*}{ 2-path } & $\mathrm{Cu}-\mathrm{O}_{\mathrm{eq}}$ & $4 *$ & 1.900 & 0.0059 & \multirow{2}{*}{0.0138} \\
\hline & & $\mathrm{Cu}-\mathrm{O}_{\mathrm{ax}}$ & $2^{*}$ & 1.955 & 0.0011 & \\
\hline $\mathrm{Cu}-\mathrm{Mt}-\mathrm{d}$ & 1-path & $\mathrm{Cu}-\mathrm{O}$ & $4 *$ & 1.941 & 0.0059 & 0.0430 \\
\hline \multirow{3}{*}{$\mathrm{NaCu}-\mathrm{Mt}-\mathrm{w}$} & 1-path & $\mathrm{Cu}-\mathrm{O}$ & 3.6 & 1.961 & 0.0046 & 0.0278 \\
\hline & 2-path & Cu-Oeq & $4 *$ & 1.976 & 0.0050 & \multirow[t]{2}{*}{0.0161} \\
\hline & & Cu-Oax & $2 *$ & 2.277 & 0.0148 & \\
\hline $\mathrm{NaCu}-\mathrm{Mt}-\mathrm{d}$ & 1-path & $\mathrm{Cu}-\mathrm{O}$ & $4 *$ & 1.942 & 0.0057 & 0.0421 \\
\hline
\end{tabular}


Table 3. $\mathrm{Cu}(\mathrm{II})$ coordination in the interlayer of montmorillonite

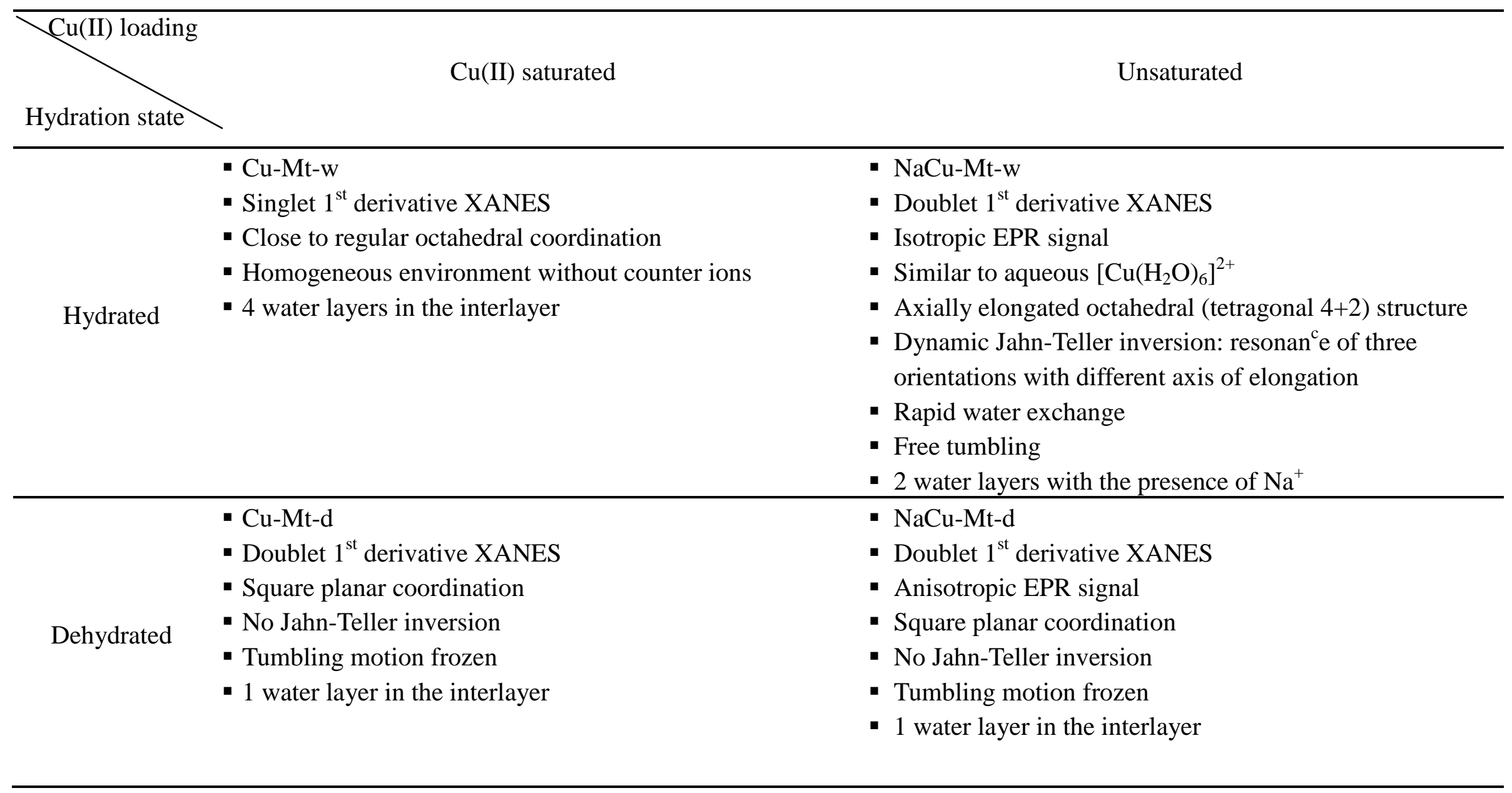




\section{Figure Captions}

Figure 1. XRD patterns of $d_{001}$-relection of wet and dry $\mathrm{Cu}$-saturated montmorillonite $(\mathrm{Cu}-\mathrm{Mt})$.

Figure 2. EPR of the interlayer $\mathrm{Cu}(\mathrm{II})$ on montmorillonite $(\mathrm{NaCu}-\mathrm{Mt})$ after evacuation at 500mTorr.

Figure 3. EPR of interlayer $\mathrm{Cu}(\mathrm{II})$ in $\mathrm{NaCu}-\mathrm{Mt}$ measured at different degree of dehydration: samples evacuated at a) 200 mTorr, b) 380 mTorr, and c) 500 mTorr, and d) simulation of 500 mTorr spectrum (c).

Figure 4. Measured $1^{\text {st }}$ derivative XANES spectra of the different $\mathrm{Cu}(\mathrm{II})$ samples and model compounds (Cu-Mt-w: $\mathrm{Cu}(\mathrm{II})$-saturated wet montmorillonite; $\mathrm{Cu}-\mathrm{Mt}-\mathrm{d}$ : $\mathrm{Cu}(\mathrm{II})$-saturated dry montmorillonite; $\mathrm{NaCu}-\mathrm{Mt}-\mathrm{w}$ : partially $\mathrm{Cu}(\mathrm{II})$-exchanged wet Na-montmorillonite; $\mathrm{NaCu}-\mathrm{Mt}-\mathrm{d}$ : partially $\mathrm{Cu}$ (II)-exchanged dry Na-montmorillonite; $\mathrm{Cu}$ PPT: $\mathrm{Cu}$ (II) oxide precipitate; Aqua $\mathrm{Cu}$ : $\mathrm{Cu}(\mathrm{II})$ aquatic solution).

Figure 5. Simulation results of the $1^{\text {st }}$ derivative XANES spectra for the different $\mathrm{Cu}$ (II) coordination.

Figure 6. Simulation results of the $1^{\text {st }}$ derivative XANES spectra for the tetragonal coordination as a function of $\mathrm{Cu}-\mathrm{O}_{\mathrm{ax}}$ distance at fixed $\mathrm{Cu}-\mathrm{O}_{\mathrm{eq}}$ distance of $1.95 \AA$. 
Figure 7. FEFF calculation of hypothetical $\mathrm{Cu}\left(\mathrm{O}_{\mathrm{eq}}\right)_{4}\left(\mathrm{O}_{\mathrm{ax}}\right)_{2}$ compounds: a) EXAFS and b) radial structure functions. $\mathrm{Cu}-\mathrm{O}_{\text {eq }}$ was fixed at $1.95 \AA$ while $\mathrm{Cu}-\mathrm{O}_{\text {ax }}$ varied at $2.10 \AA, 2.25 \AA, 2.50 \AA$, and $2.75 \AA$.

Figure 8. EXAFS data and fitting results along with component path contributions (Data: measured data, Fit: FEFF fit results, Oeq: contribution from the equatorial oxygen atoms, and Oax: contribution from the axial oxygen atoms). (a) $k^{3}$-weighted $\chi(k)$ for the fully hydrated $\mathrm{Cu}$ saturated montmorillonite (Cu-Mt-w), (b) Fourier transform magnitude for Cu-Mt-w, (c) $k^{3}$ weighted $\chi(k)$ for the fully hydrated unsaturated montmorillonite (NaCu-Mt-w), and (d) Fourier transform magnitude for NaCu-Mt-w. 


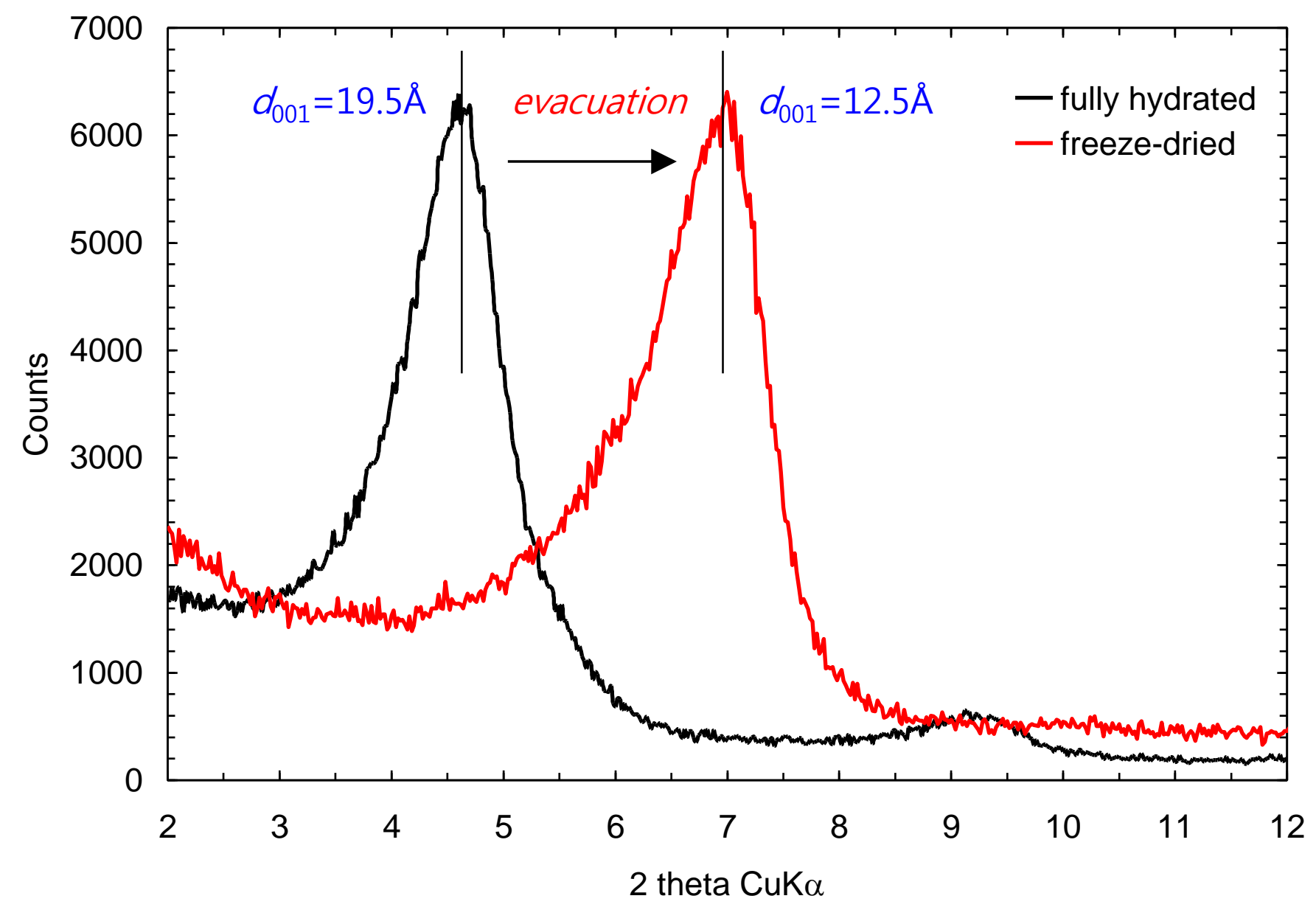


Figure 2

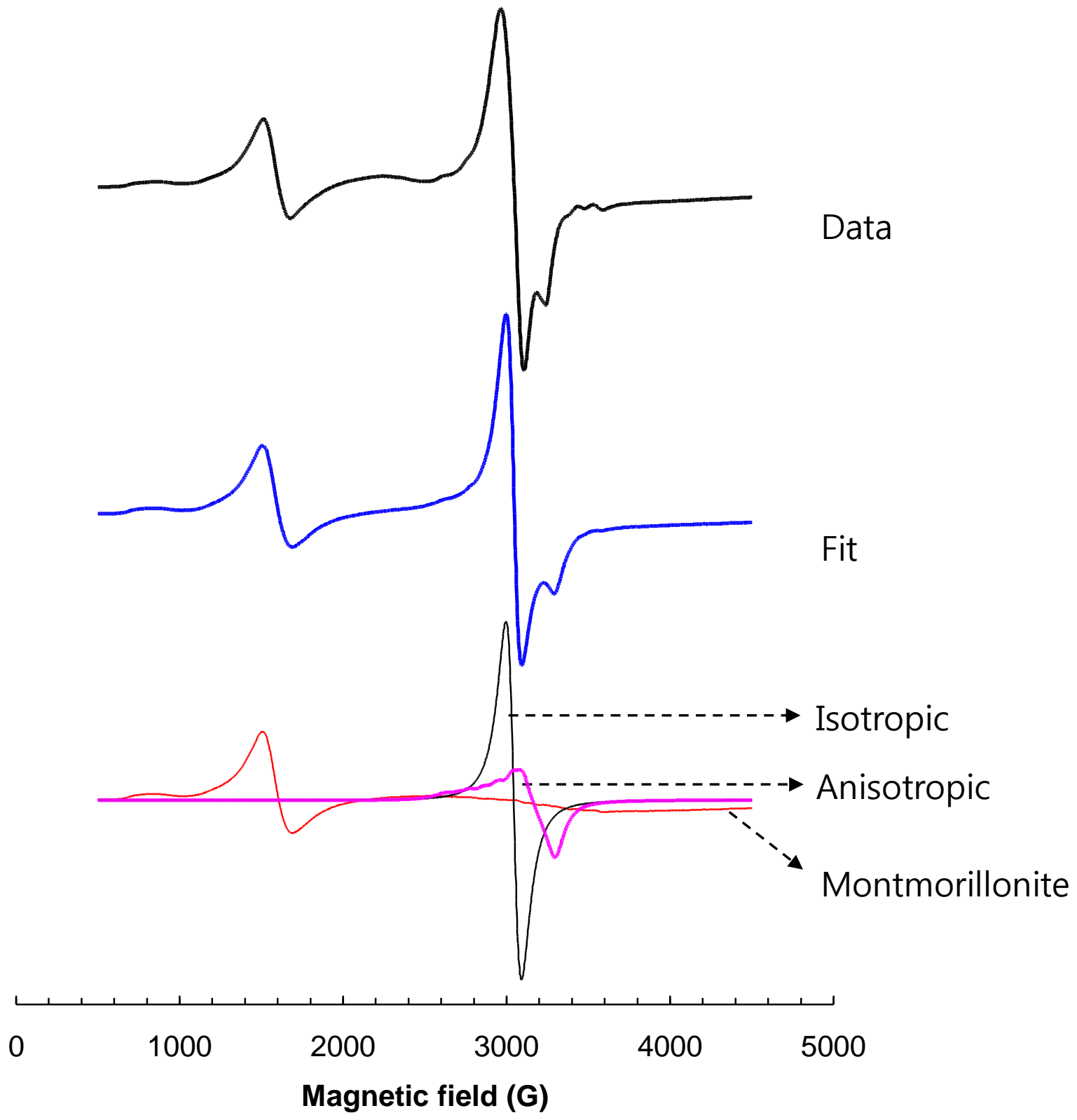


Figure 3

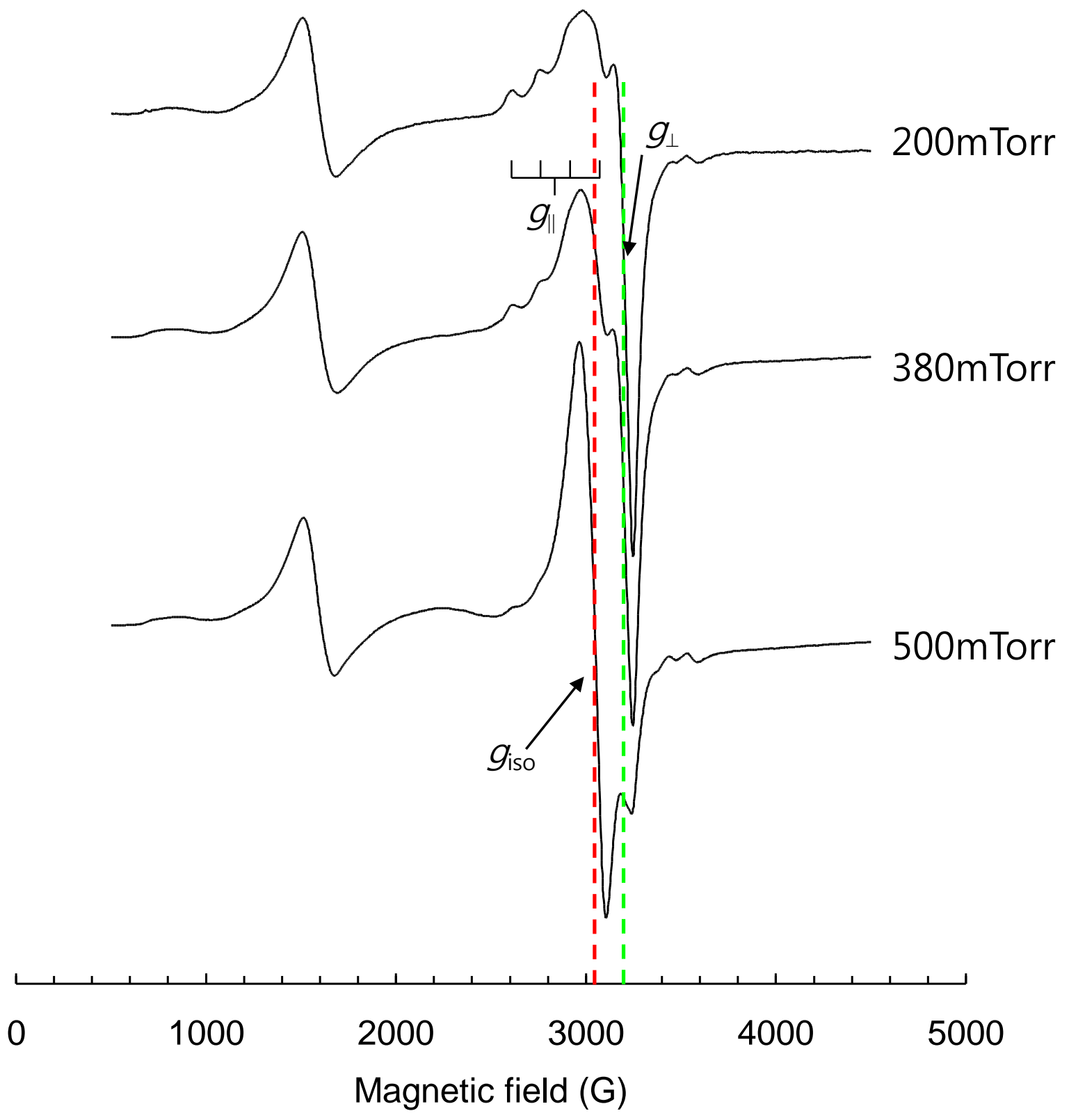




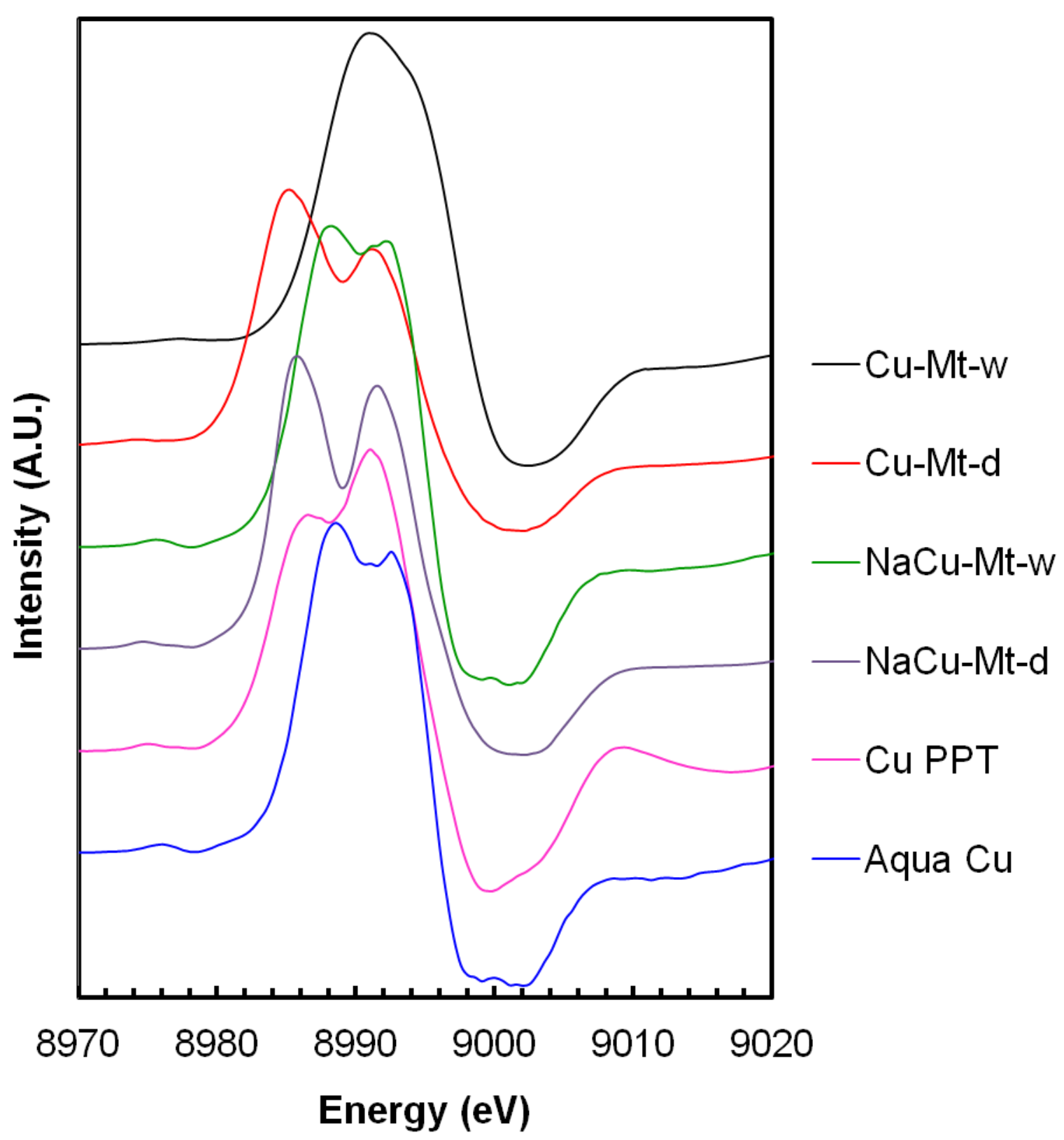


Figure 5

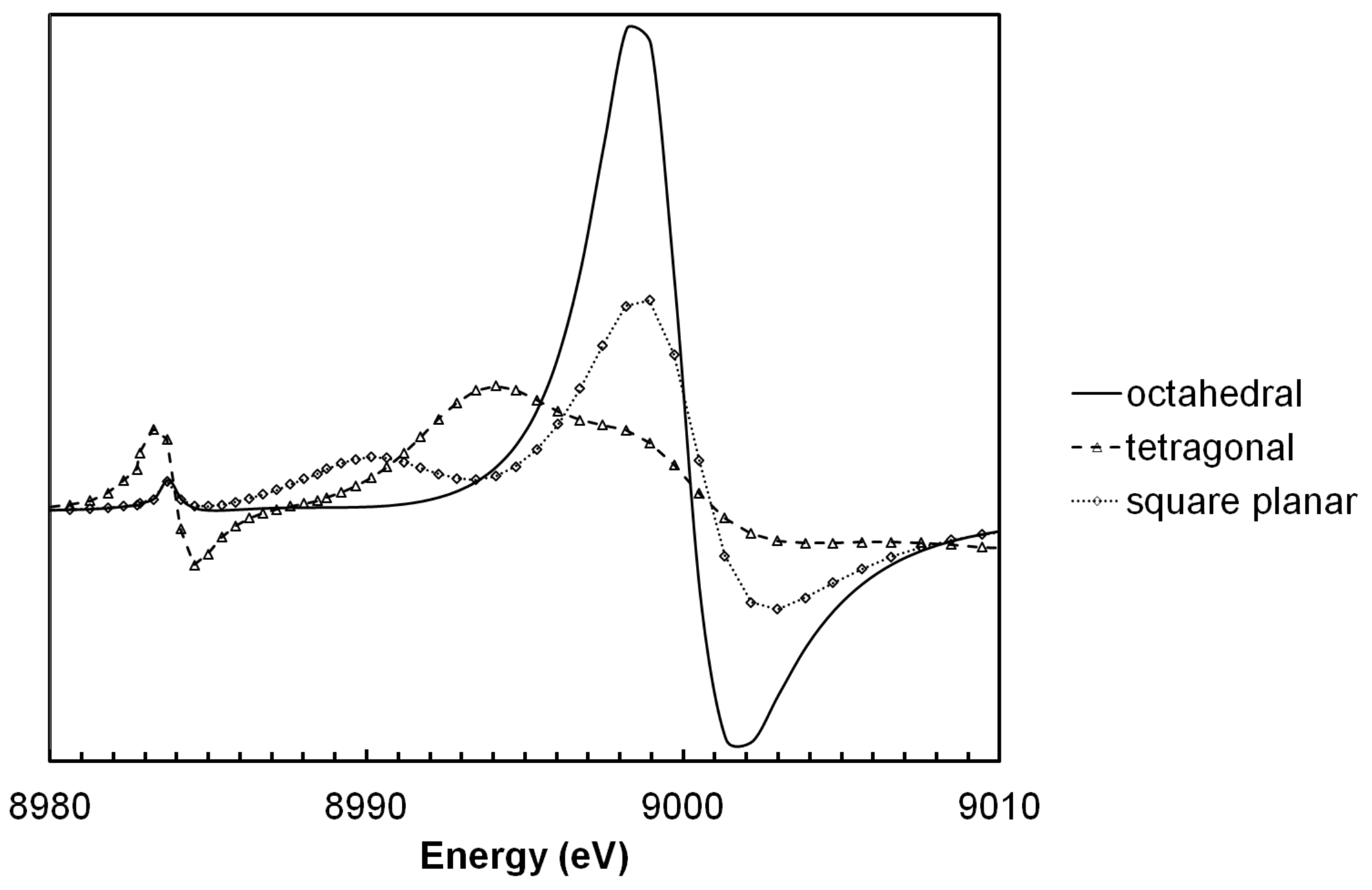


Figure 6

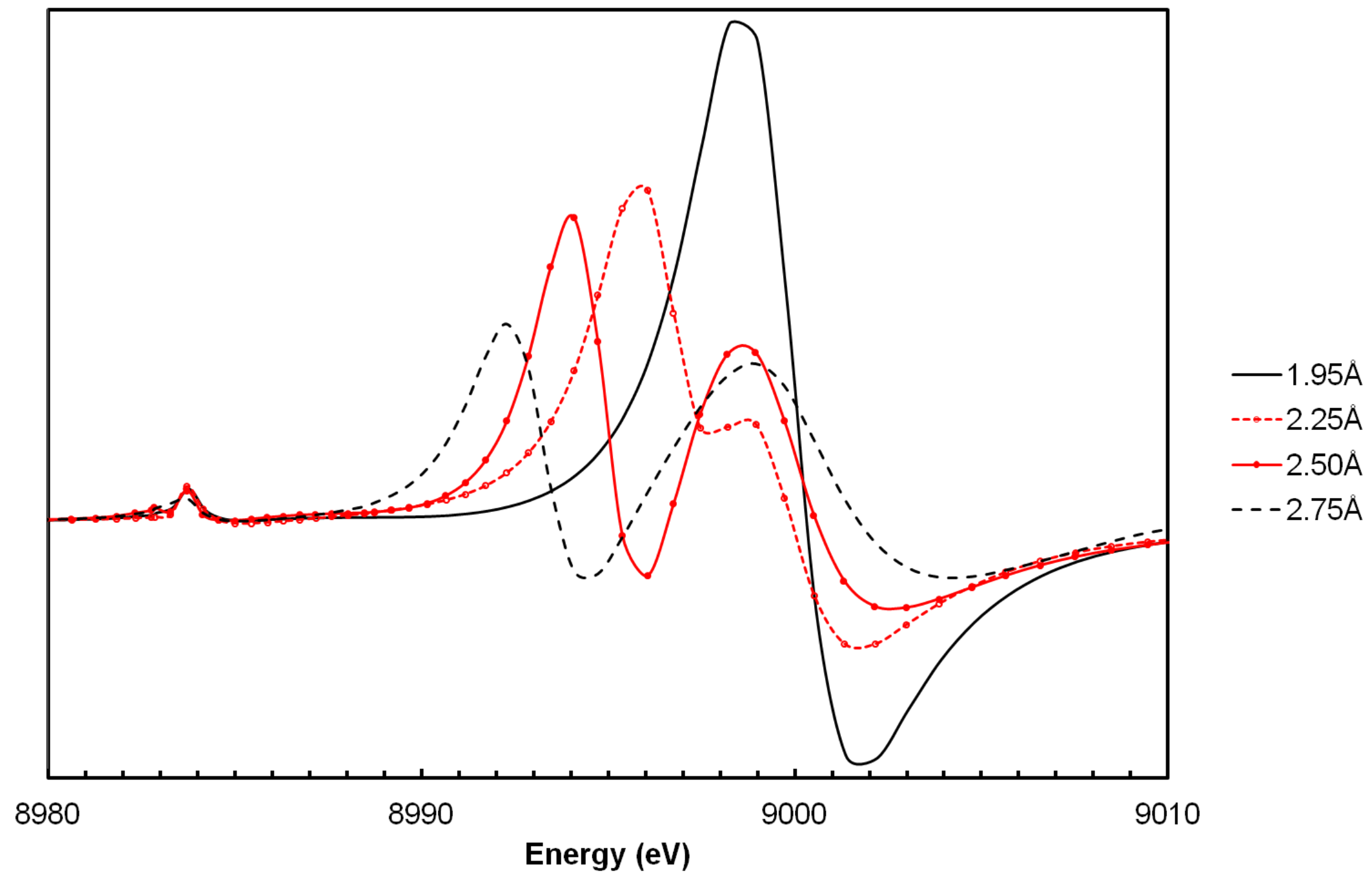


Figure 7
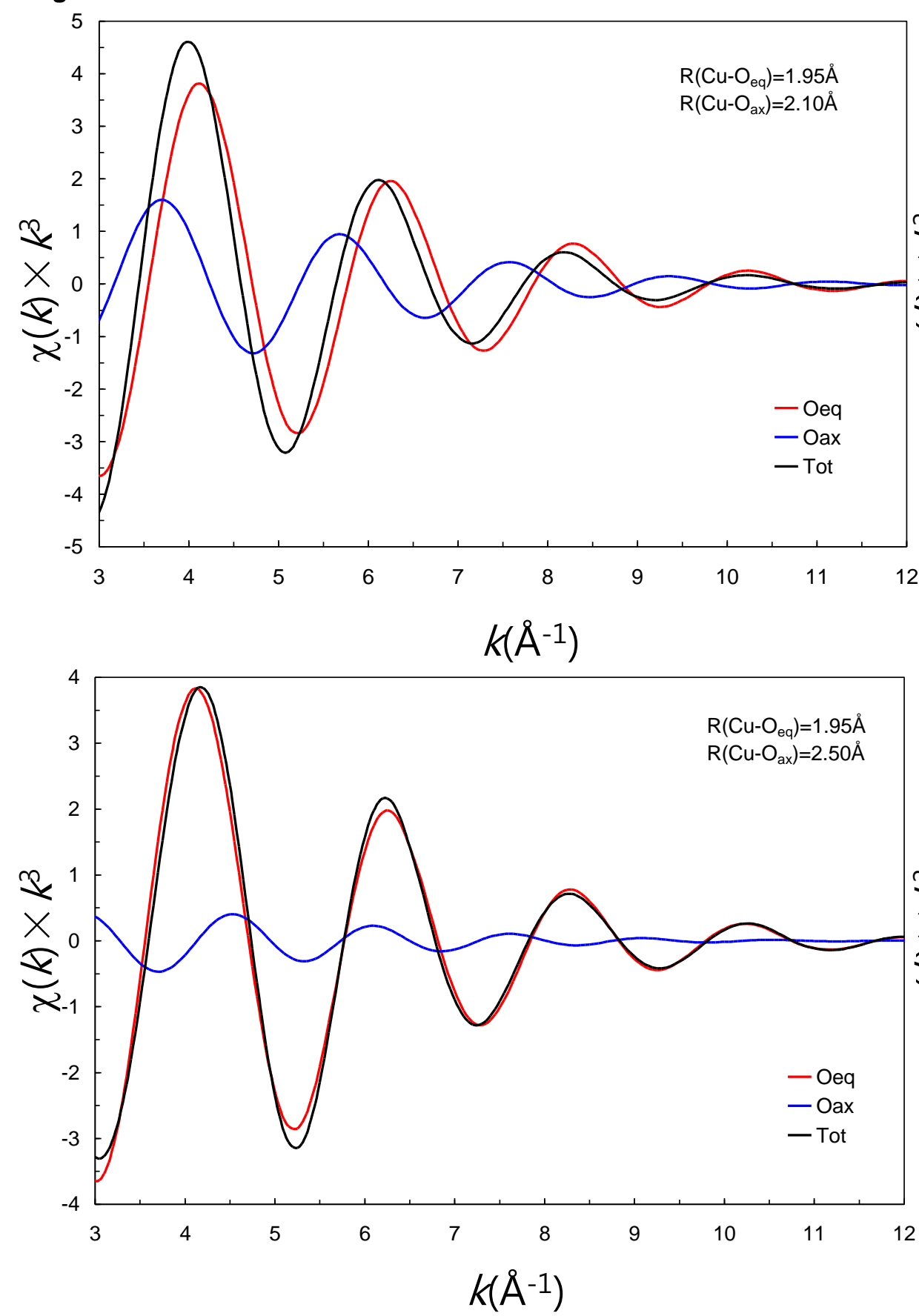
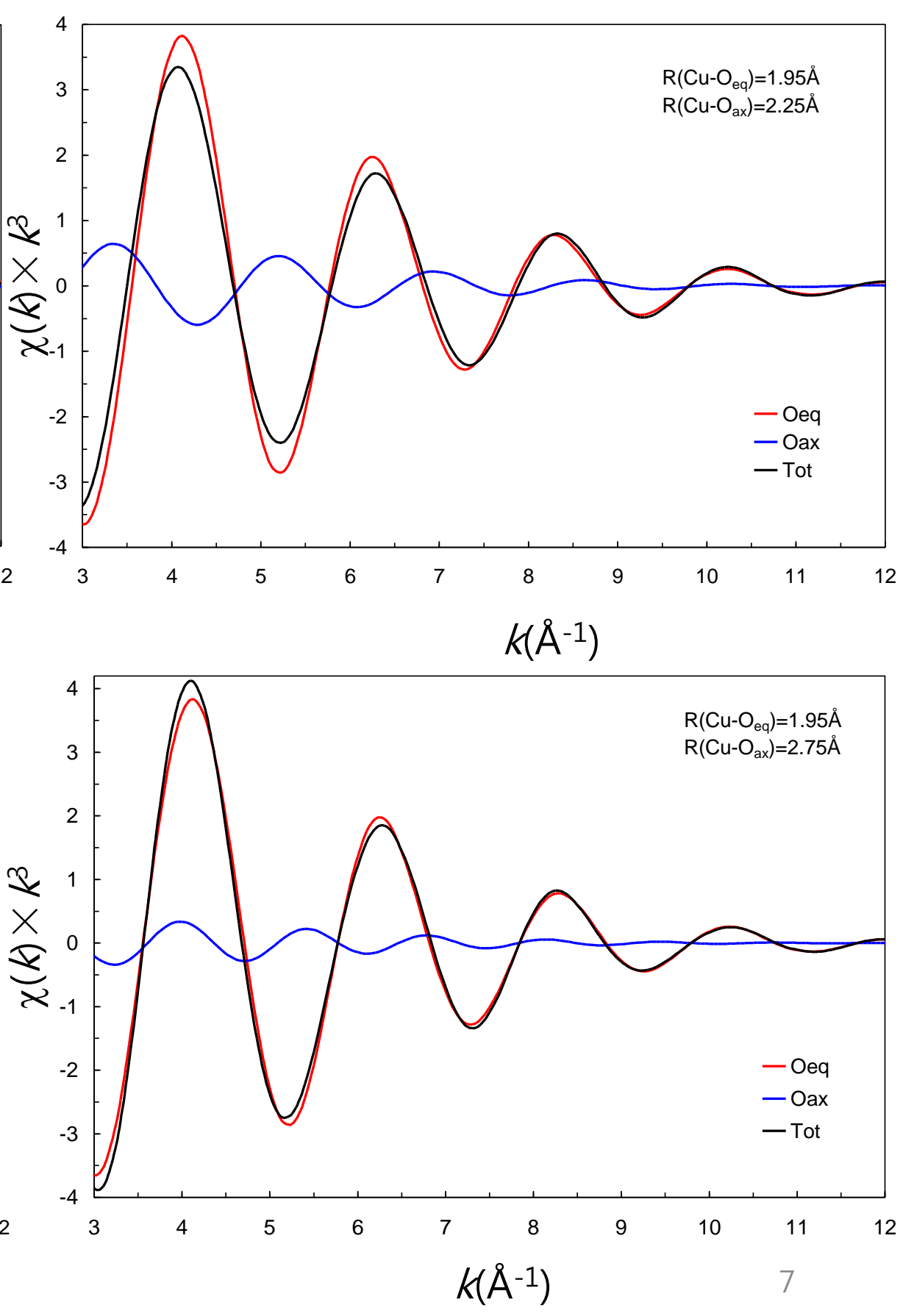
Figure 8
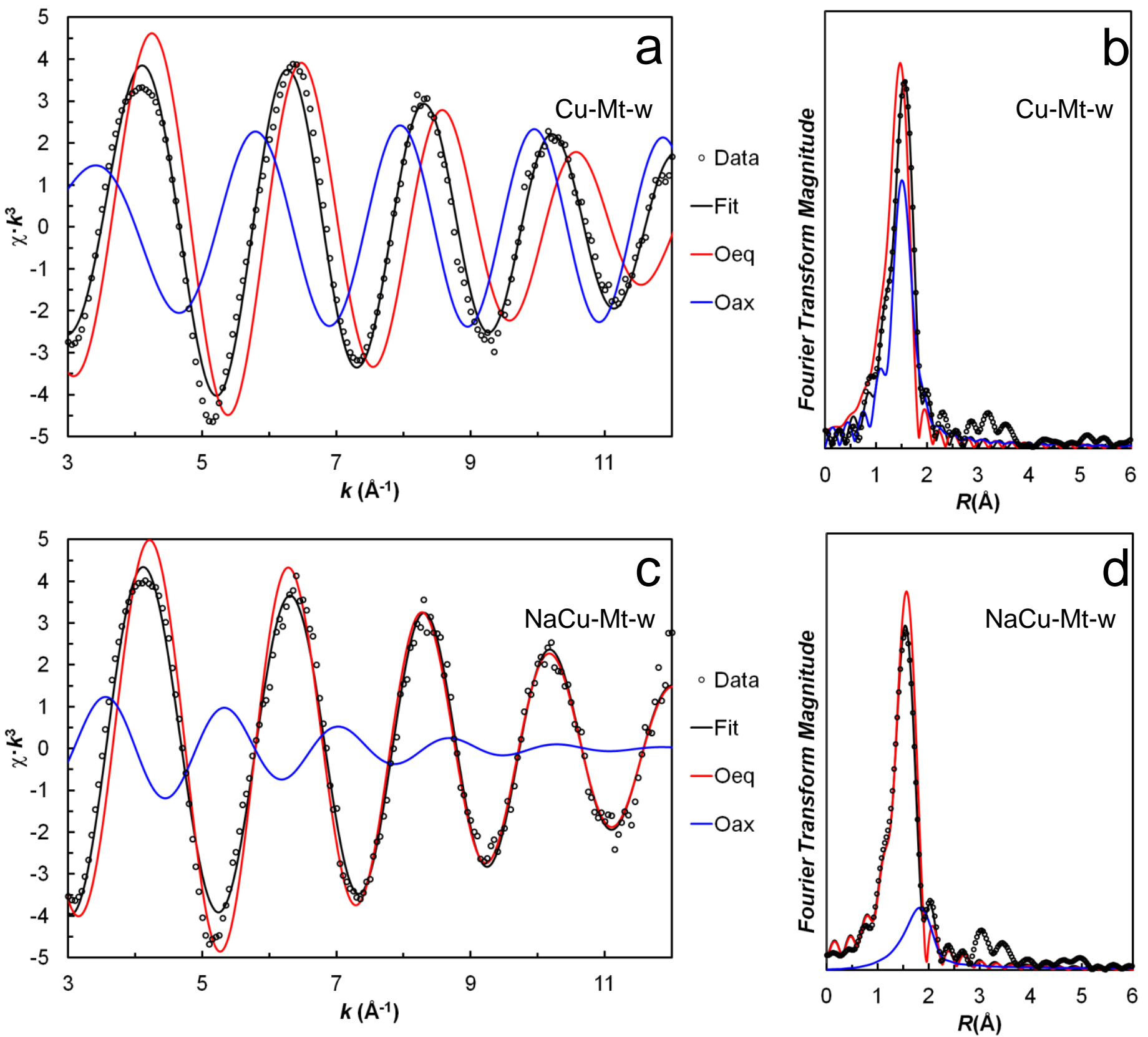